\title{
La incidencia del Impuesto Especial sobre Producción y Servicios al tabaco en México
}

\author{
Tobacco Tax Incidence in Mexico
}

\author{
Jorge Ibarra Salazar*, Daniela Patricia de la Fuente Pérez, \\ María Fernanda Miravete Martínez \\ Instituto Tecnológico y de Estudios Superiores de Monterrey, México
}

Recibido el 14 de enero de 2019; aceptado el 7 de noviembre de 2019

Disponible en Internet el: 21 de noviembre de 2019

\section{Resumen}

Los productos de tabaco en México están gravados con el IEPS y el IVA. El primero tiene un componente ad-valorem de $160 \%$ y otro específico de $\$ 0.35$ por cigarrillo. Uno de los objetivos de los impuestos es reducir el consumo de tabaco para así bajar la incidencia de enfermedades asociadas al tabaquismo. Esto depende, sin embargo, de que el impuesto sea trasladado al precio que pagan los consumidores. En este artículo estimamos la incidencia del IEPS al tabaco en México usando una base de datos mensuales de enero 1994 a junio de 2017. Encontramos que, si bien el componente ad-valorem y el específico del IEPS guardan una relación directa y estadísticamente significativa con el índice de precios de productos de tabaco, la carga del IEPS no es trasladada a los consumidores. Las modificaciones en precios se explican primordialmente por los aumentos en costos que enfrentan las empresas que fabrican productos de tabaco. Este resultado resta efectividad a la política fiscal que tiene por objeto reducir el consumo de tabaco.

Código JEL: H22, I18, L66

Palabras clave: Incidencia impositiva; Impuesto tabaco; IEPS; México

\footnotetext{
*Autor para correspondencia

Correo electrónico jaibarra@tec.mx (J. Ibarra Salazar).

La revisión por pares es responsabilidad de la Universidad Nacional Autónoma de México. 


\begin{abstract}
Tobacco products in Mexico are levied with the excise and value added taxes. The former has and ad-valorem component at $160 \%$, and a per unit component at $\$ 0.35$ per cigarette. One of the objectives of tobacco taxes is to reduce its consumption, so that tobacco related diseases are also reduced. That depends, however, of how the tax is shifted into the price paid by the consumers. Using monthly data from January 1994 to June 2017, in this paper, we estimate the incidence of the excise tobacco tax. We find that both, the ad-valorem and the specific components of the excise tax have a positive and statistically significant relationship to the tobacco price index, but the tax is not shifted to the consumers. Price changes are explained by increases in production costs faced by tobacco producers. This result diminishes the effectiveness of fiscal policy aimed to reduce tobacco consumption.
\end{abstract}

JEL codes: H22, I18, L66

Keywords: Tax incidence; Tobacco tax; IEPS; Mexico

\title{
Introducción
}

Se considera que los impuestos al tabaco son una herramienta que se puede usar para reducir el consumo de tabaco, y consecuentemente las enfermedades asociadas al tabaquismo. El Convenio Marco de la Organización Mundial de la Salud para el Control del Tabaco $(\mathrm{CMCT})^{1}$ reconoce a los impuestos como un medio eficaz e importante para reducir el consumo de tabaco. En México, también se ha reconocido que los impuestos especiales persiguen frecuentemente fines extrafiscales, más allá de la recaudación de ingresos fiscales, ya que tiene el potencial de afectar el consumo de tabaco (CEFP, 2002). En efecto, cuando se propuso aumentar la tasa del impuesto e introducir un componente específico al impuesto sobre tabacos labrados, se señalaba que dicha iniciativa, además del fin recaudatorio se pretendía contribuir al combate del tabaquismo. ${ }^{2}$

La efectividad de los impuestos al tabaco depende, sin embargo, del traslado de los impuestos a los precios pagados por los consumidores. Si el aumento en impuestos no aumenta los precios pagados por los consumidores, esta herramienta deja de ser efectiva en reducir el tabaquismo. Es entonces que la determinación de la magnitud de traslado a los precios pagados por el consumidor, ante aumentos en impuestos, es vital para determinar qué tan efectivos son los impuestos en reducir el consumo de tabaco. En México, la estructura oligopólica de la industria del tabaco, la diversidad de marcas y segmentación de mercados propias de la

\footnotetext{
${ }^{1}$ https://www.who.int/fctc/text download/es/

${ }^{2}$ Exposición de motivos para aumentar el IEPS a tabacos labrados en 2010. Disponible en: http://www.diputados. gob.mx/PEF2010/temas/expo_motivos/ingresos/iniciativa_lieps.pdf
} 
industria y la magnitud del comercio ilegal de tabaco, pueden hacer que los impuestos no sean trasladados totalmente a los precios pagados por los consumidores.

El efecto que tienen los impuestos es un asunto de importancia en la economía del tabaco, ya que puede influir en el bienestar de los fumadores y sobre quienes no consumen productos de tabaco, en las empresas que participan en la industria y también en la política pública hacia la industria. Esto, aunado al efecto sobre las utilidades de las firmas, es comparado con la recaudación para determinar la carga excesiva del impuesto, que constituye una medida de la ineficiencia que puede ser causada por la medida fiscal.

Los estudios sobre impuestos al tabaco en México han supuesto que los impuestos son trasladados completamente a los precios pagados por los consumidores. No es de nuestro conocimiento que se haya estimado la incidencia de los impuestos al tabaco.

Nuestro objetivo en este artículo es analizar la incidencia del IEPS a tabacos labrados. La incidencia se refiere a la forma en que se distribuye la carga del impuesto entre productores y consumidores en el mercado de un producto. En el caso particular que nos ocupa en este artículo, se trata de averiguar cómo afectan cambios en el IEPS a los precios del tabaco. Dicho de otra forma, tratamos de averiguar si los aumentos en el impuesto se han trasladado a los precios que pagan los consumidores por los productos de tabaco.

Para cumplir con dicho objetivo, especificamos un modelo en forma reducida y lo estimamos usando 282 observaciones mensuales (enero, 1994 a junio, 2017). En las ecuaciones, que relacionan el precio de los productos de tabaco y los impuestos, se incluyeron como variables de control los determinantes de la demanda de tabaco, los costos de producción de productos de tabaco, la estructura de mercado de la industria de tabaco, así como la tendencia y estacionalidad de los precios de tabaco.

En la perspectiva de la política pública, se espera que al trasladarse los impuestos a los precios que pagan los consumidores se reduzca la demanda de tabaco y, por tanto, las enfermedades asociadas al tabaquismo. En otras palabras, el estudio de incidencia nos permitirá evaluar la efectividad de los impuestos como una herramienta para reducir el tabaquismo.

Nuestros resultados muestran que, si bien el IEPS guarda una relación directa y estadísticamente significativa con el índice de precios de productos de tabaco, éste último es poco sensible a los cambios en los componentes ad-valorem y específico del IEPS. Los costos de producción son los que principalmente influyen en la evolución de los precios del tabaco en México.

En las siguientes secciones presentemos brevemente el estado y evolución de los impuestos al tabaco en México, comentamos la literatura sobre incidencia de impuestos al tabaco, presentamos la metodología, los resultados y finalmente nuestras conclusiones. 


\section{Tabaquismo y los impuestos al tabaco en México}

El Instituto Nacional de Salud Pública, en la exposición del panorama epidemiológico asociado al tabaquismo (INSP, 2011), anota que el consumo de tabaco y la exposición al humo de tabaco son la primera causa de muerte que se puede prevenir a escala mundial (Kuri et al.,2006). Las encuestas nacionales sobre adicciones evidencian que el tabaquismo en México es un problema de salud pública especialmente entre adolescentes (Reynales et al., 2009), adultos jóvenes y mujeres (Reynales et al., 2011). De acuerdo con la Secretaría de Salud (SSA, 2011), dentro de las diez primeras causas de mortalidad se encuentran las enfermedades relacionadas con el tabaquismo y la exposición al humo de tabaco (infarto agudo al miocardio, enfermedades cerebro - vasculares, respiratorias crónicas y cáncer de pulmón). Como consecuencia, se ha documentado que el consumo de tabaco ocasiona más de 60 mil muertes al año (Sáenz de Miera et al., 2012); que afecta en forma negativa a la economía familiar (World Bank, 1999); que reduce la productividad laboral (Guerrero et al., 2012); y que impone costos importantes al sector salud (Reynales et al., 2006).

La Encuesta Nacional de Adicciones 2011 reporta una prevalencia de consumo activo de tabaco de $21.7 \%$ (17.3 millones de personas): 12 millones son hombres y 5.3 son mujeres. El 26.4\% (21 millones) reportaron ser exfumadores, 20.1\% ser exfumadores ocasionales y el 51.9\% (41.3 millones de personas) de la población nunca ha fumado. Se encontró también que, en promedio, los fumadores diarios entre 12 y 65 años inician el consumo diario de tabaco a los 20.4 años de edad; y que fuman en promedio 6.5 cigarros al día. El $11.4 \%$ de los fumadores activos (1.5 millones de personas) encienden su primer cigarro 30 minutos después de despertar, y de acuerdo con este criterio son considerados como adictos a la nicotina. También se reporta que el 30.2\% (12.5 millones de personas) nunca ha fumado y se encuentra expuesta a humo de tabaco ambiental (HTA).

La CONADIC (2011), después de presentar los resultados del sistema de monitoreo del tabaquismo en México, concluye, entre otros aspectos que: si no se revierte la tendencia del tabaquismo, la mitad de los fumadores activos (17.3 millones) morirán a causa de enfermedades relacionadas con el tabaco; cada año mueren 60 mil personas por causas atribuibles al tabaco; el problema del tabaquismo en jóvenes representa un reto para la salud pública de México. El gran reto, anota la CONADIC, es implementar políticas públicas para detener las tendencias observadas, ya que la epidemia de los padecimientos asociados al consumo de tabaco para los siguientes 20 o 30 años representará una carga que el Sistema Nacional de Salud difícilmente podrá solventar.

En respuesta a la problemática del tabaquismo a escala nacional, México firmó y ratificó el CMCT en 2003, que fue publicado en el DOF en mayo de 2004. Este convenio es un tratado mundial que contiene las bases para aplicar y gestionar programas de control del consumo 
y la producción de tabaco. Consecuentemente, se promulgó en México la Ley General para el Control del Tabaco, fortaleciéndose el aparato institucional para aplicar la regulación correspondiente. Con la suscripción del CMCT se han implementación un conjunto de medidas regulatorias hacia la industria en México. Por el lado de la demanda, se ha adoptado una política fiscal más agresiva combinando impuestos específicos y ad-valorem; se ha restringido la publicidad de productos de tabaco; se ha regulado el etiquetado y empaque de cigarrillos para incluir leyendas y pictogramas; existe un sistema de programas de prevención; se han adoptado medidas para proteger a los no fumadores; y medidas también para restringir la oferta (CONADIC, 2011; COFEMER, 2012).

Los cigarrillos en México están gravados con el IEPS y con el Impuesto al Valor Agregado (IVA). El IEPS puede ser específico o ad-valorem. En el primer caso, se carga un impuesto fijo por unidad de producto al fabricante, productor o importador. En el segundo, se cobra un impuesto como porcentaje sobre el precio de la producción al fabricante, productor o importador. Por otro lado, el IVA es trasladado en la cadena de producción hasta el consumidor final $^{3}$. El proceso es entonces el siguiente: primero se agrega el IEPS al precio al detallista; luego el detallista le suma su ganancia y costos, lo cual equivale a alrededor de un $10.72 \%$ del precio al detallista después del IEPS, y finalmente se añade el IVA al precio de venta al público. Además del IEPS y el IVA, los productos de tabaco importados pagan otro impuesto ad-valorem. La tasa vigente para los cigarros importados es de $67 \%$ del precio al importador (Sáenz de Miera, 2013).

Actualmente, la tasa de IEPS a los cigarrillos es de $160 \%$ sobre el precio de venta al detallista y el IEPS específico es de $\$ 0.35$ pesos por cigarro. La tasa de IVA es de $16 \%$ sobre el precio de venta al consumidor. La última vez que se modificaron los impuestos a los cigarros fue en 2011. Tal como se muestra en la figura 1, el precio de venta al público de una cajetilla de cigarros se integraba de la siguiente manera: el precio del mayorista, que incluye el precio de fábrica, la ganancia y gastos generales del mayorista, representa un $22.86 \%$; el IEPS ad-valorem un $36.58 \%$ y el componente especifico de este impuesto un $18.42 \%$; un $8.35 \%$ corresponde a las ganancias y gastos generales del minorista; y el IVA representa un $13.79 \%$.

\footnotetext{
${ }^{3} \mathrm{http}: / /$ www.sat.gob.mx/informacion_fiscal/preguntas_frecuentes/Paginas/ieps.aspx http://www.sat.gob.mx/informacion_fiscal/obligaciones_fiscales/personas_morales/no_lucrativas/Paginas/concepto_iva.aspx
} 


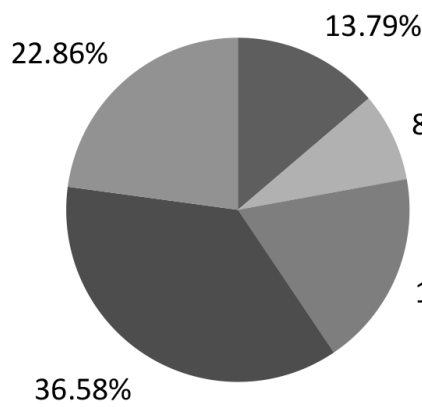

- IVA

$8.35 \%$

Ganancia y gastos

generales del minorista

IEPS específico

$18.42 \%$

- IEPS ad valorem

Figura. 1. Composición del precio de una cajetilla de cigarros en México (2011).

Fuente: elaboración propia con datos de COFEMER (2012).

La tabla 1, compara la recaudación por concepto de IEPS de tabacos labrados con la de otros impuestos federales. En el año 2016, el ingreso tributario por concepto de IEPS a tabacos labrados fue de \$38 mil millones de pesos (mmp). Esta cantidad representó un 9.2\% de la recaudación total de IEPS, un 4.8 de la recaudación del IVA y un $2.7 \%$ de la recaudación del impuesto sobre la renta (ISR).

Tabla 1

Ingresos tributarios por IEPS a tabacos labrados con respecto a otros impuestos, 2016 (millones de pesos)

\begin{tabular}{lcc}
\hline & Recaudación & Proporción \\
\hline Impuesto Sobre la Renta & $\$ 1425794.3$ & $2.670 \%$ \\
Impuesto al Valor Agregado & 791700.20 & $4.812 \%$ \\
IEPS total & 411389.60 & $9.261 \%$ \\
IEPS tabacos labrados & 38097.10 & \\
\hline
\end{tabular}

Fuente: elaboración propia con datos de la SHCP (Ingresos Presupuestarios del Gobierno Federal, www.shcp.gob.mx)

El IEPS a tabacos labrados entró en vigor en 1981 con una tasa de 139.4\%. La tasa más alta de este impuesto se registró durante 1986-87 (180\%), para a partir de entonces reducirse hasta llegar al 85\% en 1995-99. A partir del año 2000, la tasa del IEPS se ha elevado hasta llegar a 160\%, y se mantiene en ese nivel desde 2011 (figura 2). ${ }^{4}$

\footnotetext{
${ }^{4}$ CEFP (2002), Sáenz de Miera, et al. (2013) y Sáenz de Miera e Iglesias (2010) estudian la evolución de los impuestos al tabaco en México. CEFP (2018) describe el marco jurídico y los efectos fiscales de la industria del tabaco.
} 


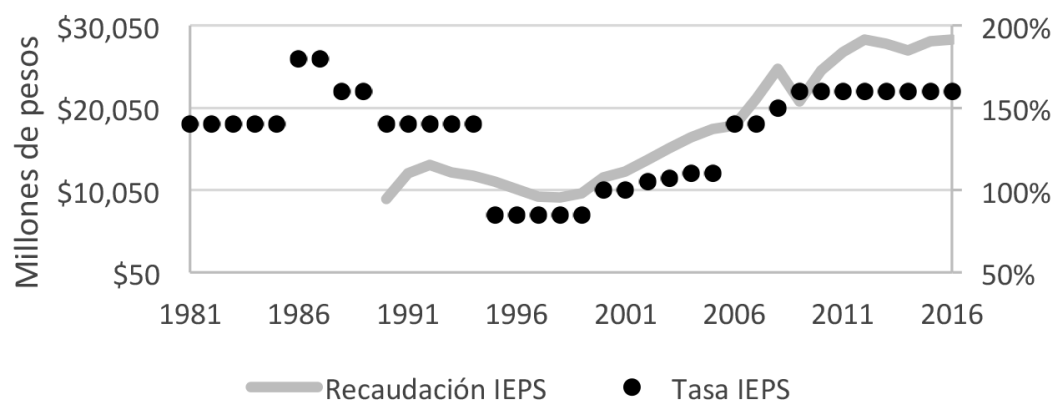

Figura. 2. Evolución de la recaudación de IEPS a tabacos labrados (millones de pesos de 2008) y tasa de IEPS de tabacos labrados con filtro (porcentajes), 1981-2016.

Fuente: elaboración propia con datos de SHCP (Ingresos presupuestarios del gobierno federal, www.shxp.gob.mx), Ramírez-Barba et al. (2008), Sáenz de Miera (2013) e INSP (2012).

Entre las explicaciones que se han brindado para entender las variaciones en la tasa del IEPS a tabacos labrados, Sáenz de Miera e Iglesias (2010) y Sáenz de Miera (2013) atribuyen el aumento de esa tasa a $180 \%$, en 1986, a la necesidad de mejorar la recaudación fiscal, en ese año de crisis económica severa. Por otro lado, Meneses González et al. (2002) sugieren que, aunado a otras acciones, la baja de la tasa del IEPS observada en la década de los 90's reduciría el contrabando de tabaco. Al revisar la evolución del impuesto al tabaco, Sáenz de Miera (2013: p. 144) anota que "este impuesto ha sufrido varios cambios desde su creación en 1981, pero sólo hasta hace alrededor de diez años comenzaron a vincularse claramente con fines de salud pública." Esto último, es coincidente con la firma y ratificación del CMCT por parte de México.

Es interesante notar que, diferentes estudios argumentan que detrás de los cambios en la tasa del IEPS a tabacos están también los grupos de interés. En particular, Madrazo Lajous y Guerrero Alcántara (2012: p. 316) sugieren que las empresas que conforman la industria del tabaco pueden influir en la política fiscal hacia la industria. Refieren, por ejemplo, un acuerdo firmado en 2004, entre el gobierno federal y la industria, en que se "establecía el acuerdo común de evitar la imposición de nuevos o mayores impuestos al tabaco.” De acuerdo con estos autores, la industria busca que "los impuestos se mantengan en el nivel más bajo posible como porcentaje del precio final al consumidor o, en su defecto, que el aumento en impuestos sea gradual y no sea ad-valorem." Ochoa (2013), explica la labor de las organizaciones de la sociedad civil en el proceso, que, durante 2009 condujo al aumento de la tasa del IEPS a $160 \%$ y a la fijación del componente específico de dicho impuesto. 
Aunado a la reducción en la tasa de IEPS a mediados de los noventas, las condiciones económicas contribuyeron a la reducción real de la recaudación del IEPS hacia fines de la década de los 90's. Si bien en términos nominales, la recaudación de este impuesto pasó de \$2 407 mdp en 1992 a \$4 990 en 1998, al considerar la inflación resultó que la recaudación se redujo un $30 \%$ en términos reales: una caída de $4.3 \%$ promedio anual. En la década de 1998 a 2008, la recaudación del IEPS mostró un crecimiento sostenido, al pasar de \$4 990 mdp a \$24 762 mdp. Esto significó un crecimiento de casi $170 \%$ en términos reales: un $15.4 \%$ promedio anual. Este período estuvo asociado con el aumento de la tasa del IEPS y con un crecimiento moderado del ingreso promedio de los consumidores. Posteriormente, debido a la crisis financiera, la recaudación del IEPS a tabacos sufrió una reducción de $16 \%$ real en 2009, para recuperarse en los años posteriores hasta llegar a los \$38 097 mdp en 2016, tal como lo reportamos en el cuadro 1 y en la figura 2 .

El poder ejecutivo. propuso aplicar una cuota fija a los productos de tabaco, dependiendo de su gramaje, para incrementar el precio de todas las marcas y/o presentaciones en forma progresiva.

También se propuso incluir un código de seguridad en cada cajetilla para evitar el contrabando. Después de varias modificaciones de esta propuesta por parte de la Comisión de Hacienda de la Cámara de Diputados, así como de la Comisión de Hacienda del Senado, se aprobó una reforma para incluir una cuota de diez centavos por cigarro (0.75 gramos), aunque sería paulatina, empezando en 4, 6 y 8 centavos por cigarrillo en 2010, 2011 y 2012, respectivamente.

En 2010, se propuso un aumento gradual del impuesto específico de $\$ 0.04$ a $\$ 0.40$ por cigarro, así como otros ajustes al IEPS ad-valorem. Los aumentos al IEPS ad-valorem no fueron aprobados, ya que la Comisión de Hacienda de la Cámara de Diputados consideró que fomentarían la sustitución a productos de tabaco de menor precio, haciendo su efecto poco efectivo. La reforma aprobada consistió en el aumento de la cuota fija, de \$0.04 a \$0.35 pesos por cigarro, a partir de 2011. La figura 3 muestra la evolución real del componente específico en pesos por cajetilla de 20 cigarrillos. 


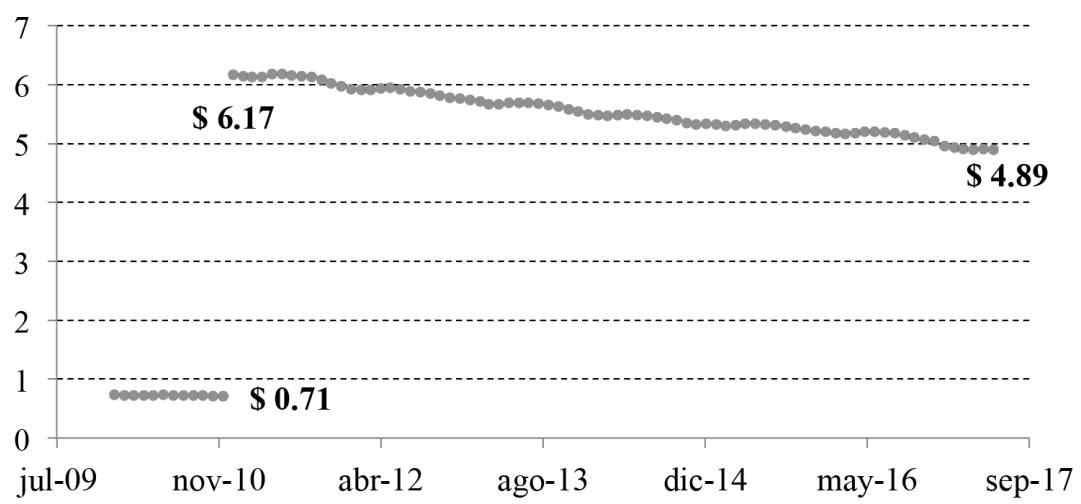

Figura. 3. Evolución del IEPS específico para tabacos labrados, enero 2010-julio 2017 (pesos reales 2010). Fuente: elaboración propia con datos de Ramírez-Barba et al. (2008), Sáenz de Miera (2013) e INSP (2012).

La figura 4 muestra el comportamiento trimestral del consumo per cápita de cigarros (C) en México y del índice de precios de productos de tabaco. Además, hemos incluido la tasa del IEPS a tabacos labrados. Como se puede observar en esa figura, aunado a un crecimiento permanente en los precios del tabaco, el consumo de tabaco se mantuvo en una banda de variación entre 110 y 160 cigarros per cápita, entre el primer trimestre de 1994 y el tercer trimestre del 2005. A partir de ese trimestre, y después de un repunte en el primer trimestre de 2006 en el que alcanzó su máximo de 191 cigarros per cápita en el período considerado, el consumo muestra una tendencia decreciente un tanto irregular. El consumo per cápita pasó del máximo histórico de 191 en el primer trimestre del 2006 a 80 hacia la mitad de $2013^{5}$. Es interesante notar la tendencia creciente que muestra el índice de precios de productos de tabaco, a pesar de que la tasa del IEPS se hubiera reducido de $150 \%$ a $85 \%$ en 1995 . Por otro lado, es notable el aumento en dicho índice de un 25\% en el primer trimestre de 2011, ya que a pesar de que la tasa del impuesto se mantuvo en $160 \%$, se ajustó el componente específico del IESP a tabacos labrados, tal como se expuso en párrafos anteriores. Controlando por otras variables que pueden influir en los precios de productos de tabaco, el objetivo de este artículo es, precisamente, determinar qué tan importante ha sido la evolución de ambos componentes del IEPS a tabacos labrados para explicar el comportamiento de los precios de productos de tabaco.

${ }^{5}$ Para un análisis de los determinantes de la demanda de tabaco, utilizando series de tiempo, se recomienda Ibarra Salazar et al. (2019) y Oliviera Chávez et al. (2010). 


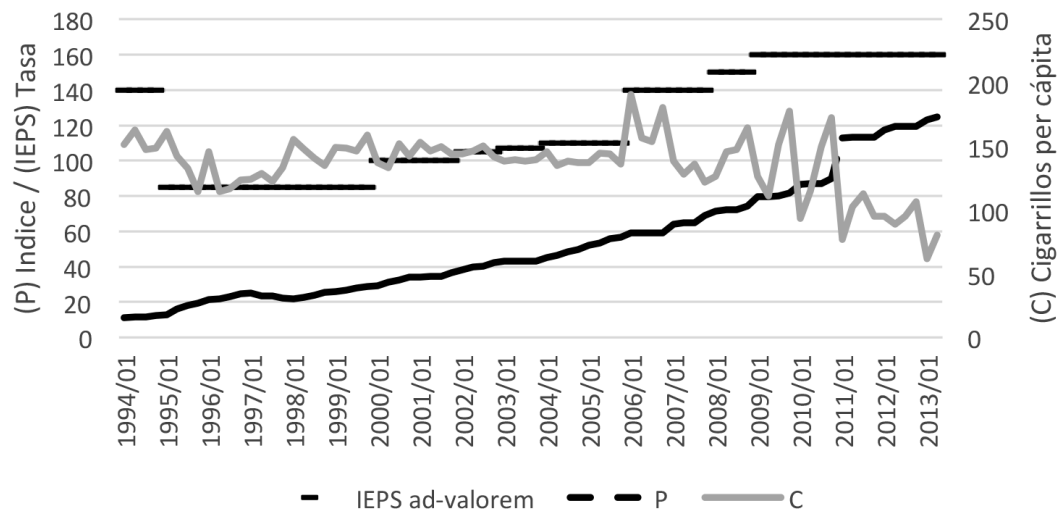

Figura. 4. Evolución del índice de precios de los productos de tabaco (P), el IEPS ad-valorem (porcentaje) y el consumo de productos de tabaco (C, cigarrillos per-cápita)6. Primer trimestre 1994-Segundo trimestre 2013 (Datos trimestrales).

Fuente: elaboración propia con datos de Ramírez-Barba et al. (2008), Sáenz de Miera (2013), INSP (2012), Olivera et al. (2010), Ibarra Salazar et al. (2019), Sistema de información arancelaria de la Secretaría de Economía (http://200.77.231.38/siavi4/fraccion.php)

y Encuesta Mensual de la Industria Manufacturara de INEGI (www.inegi.gob.mx)

A pesar de la importancia que reviste la política fiscal hacia la industria del tabaco, tanto en términos extrafiscales como fiscales, la literatura sobre el tema en México es escasa. Entre los estudios existentes, Sáenz de Miera (2013) observa que se ha avanzado en la implementación de una política fiscal más agresiva. Considera que los incrementos en impuestos han repercutido en los precios de venta al consumidor final. Argumenta que el ajuste más reciente, condujo a un incremento del precio de venta de alrededor de $25 \%$. Además, considera que la reducción del consumo pudo haber sido de un $13 \%$, toda vez que la elasticidad precio de la demanda es -0.52 .

Waters, et al. (2010), realizan simulaciones que cuantifican las reducciones proyectadas en la prevalencia del consumo, la mortalidad prematura y los aumentos en los ingresos del gobierno generados según diferentes combinaciones de impuestos. Suponiendo una elasticidad

\footnotetext{
${ }^{6} \mathrm{El}$ consumo de productos de tabaco se calculó sumando la producción nacional de tabaco y las importaciones netas de tabaco La información sobre la producción nacional para los años de 1994 a 2004 toma en cuenta únicamente la información de los cigarrillos con filtro, debido a que de 2001 a 2005 no se publicó información relacionada con los cigarrillos sin filtro. Para los años de 2005 a 2013, la variable comprende los cigarros de todo tipo. Debido a que la información sobre la producción nacional viene expresada en cajetillas, mientras que la relacionada con importaciones netas viene expresada en kilogramos, fue necesario aplicar un factor de conversión para uniformizar las unidades de medición. Una cajetilla equivale a 20 cigarros y un kilogramo equivale a 800 cigarros.
} 
precio de la demanda igual a -0.52 , su simulación base indica que ajustando el componente ad-valorem y el específico del IEPS de acuerdo con la inflación, para que juntos integren un $75 \%$ del precio final por cajetilla ${ }^{7}$, haría que el número de fumadores disminuyera en 2.58 millones y se evitarían un aproximado de 903000 muertes.

Usando datos de la Encuesta Nacional de Ingresos y Gastos de los Hogares (ENIGH) de 1994 a 2005, Jiménez et al. (2008) estiman que si los impuestos sobre los cigarrillos aumentan un $10 \%$ (porcentaje sobre el precio), entonces el precio aumentaría un $12.4 \%$, reduciría el consumo en un $6.4 \%$ y aumentaría en casi $16 \%$ el ingreso fiscal del impuesto al tabaco.

COFEMER (2012), considera la alternativa de incrementar los impuestos al tabaco en México para que representen el $75 \%$ del precio de venta al público. De acuerdo con sus estimaciones, esto reduciría el consumo en $19.3 \%$ y aumentaría la recaudación en $20.6 \%$.

\section{Los estudios sobre incidencia}

Los estudios sobre la política fiscal hacia la industria del tabaco coinciden en afirmar que los impuestos al tabaco son una de las herramientas de política más efectivas para reducir el tabaquismo, y por ende los padecimientos a la salud causados por el tabaquismo (WHO, 2015). La fijación de impuestos al tabaco tiene una gama de asuntos que son objeto de análisis en la perspectiva económica. Si bien la literatura es muy extensa, los asuntos vinculados con los impuestos están contenidos, entre otros trabajos, en el capítulo 4 de U.S. National Cancer Institute y World Health Organization (2016), en el capítulo 11 de Samet y Yoon (2010), Chaloupka y Warner (2001), y en Chaloupka et al. (2000). Con respecto a la visión de la Organización Mundial de la Salud, el reporte WHO (2015) se centra en el manejo de los impuestos para contener el tabaquismo.

Sobre la incidencia del impuesto al tabaco, estos tratados generales de la economía del tabaco, coinciden en que el traslado de los impuestos a los precios pagados por los consumidores dependen de una serie de factores: el tipo de impuesto, la naturaleza adictiva del tabaco, la estructura de mercado de la industria, la diversidad de marcas y segmentación de mercados propias de la industria, la magnitud del comercio ilegal de tabaco, la magnitud de la elusión de impuestos, entre otras estrategias que pueden usar los consumidores para eludir los impuestos.

Las bases de la literatura empírica, relacionada con la incidencia impositiva en la industria del tabaco, provienen, entre otros, de los estudios seminales de Poterba (1996) y Besley y Rosen (1999). El tema fundamental consiste en averiguar si los impuestos a los productos son trasladados completamente a los precios pagados por los consumidores. Esto responde a una pregunta, también fundamental en las finanzas públicas, conocida como incidencia

${ }^{7}$ Es la marca recomendada por la OMS (WHO, 2015). 
impositiva, que consiste en averiguar cómo se distribuye la carga de los impuestos entre los agentes participantes en los mercados.

Los trabajos que han abordado la incidencia impositiva en la industria del tabaco se pueden dividir de acuerdo a diferentes criterios. Las dimensiones de la incidencia del impuesto al tabaco, el tipo de datos que han empleado (datos agregados en serie de tiempo, panel de datos, microdatos), el enfoque para estimar el efecto de los impuestos (estructural o forma reducida) y el resultado que han encontrado con relación al traslado del impuesto a los consumidores de cigarrillos.

Con relación a las dimensiones que pueden hacer que la incidencia impositiva no sea uniforme, se han considerado diferencias regionales o geográficas (Chiou y Muehlegger, 2014; Delipalla y O'Donnell, 2001; Harding et al., 2012), las marcas de los cigarrillos (Cevik, 2016; Brock et al., 2015), las características de los cigarrillos - premium, económico, precio bajo, filtro, regular, mentolado, light - (Wang et al., 2015; Xu et al., 2014; Chiou y Muehlegger, 2014; Espinosa y Evans, 2013; Gilmore et al., 2013; Sullivan y Dutkowsky, 2012; Hanson y Sullivan, 2009), los canales de distribución - cadena comercial, comercio independiente, internet - (Brock et al., 2015; Pesko et al., 2013; Hanson y Sullivan, 2009), las características de los consumidores - ingreso y educación - (Harding et al., 2012), y el tipo de empaque de los cigarros - paquete vs cajetilla - (Wang et al., 2015; Chiou y Muehlegger, 2014; Xu et al., 2014; Pesko et al., 2013).

En cuanto al traslado del impuesto a los precios, los resultados son variados. Ashenfelter y Sullivan (1987), y Delipalla y O'Donnell (2001) encontraron que los aumentos en impuestos no necesariamente conducen a aumentos en los precios. Los estudios de Sumner y Ward (1981), Harding et al. (2012), Pesko et al. (2013), Chiou y Muehlegger (2014), y Cevik (2016) encontraron que los precios de los cigarrillos aumentan menos que el impuesto; en tanto que Sumner y Wolgenant (1985), DeCicca et al. (2013), y Espinosa y Evans (2013), muestran evidencia de que, en general, el impuesto al tabaco es completamente trasladado a los consumidores. Los resultados de Sumner (1981), Harris (1987), Sung et al. (1994), Barnett et al. (1995), Keeler et al. (1996), Hanson y Sullivan (2009), Sullivan y Dutkowsky (2012), Gilmore et al. (2013), Xu et al. (2014), Brock et al. (2015) y Wang et al. (2015) muestran evidencia de que el precio de los cigarrillos aumentó más que el impuesto (over-shifting). Tal como hemos comentado arriba, Xu et al (2014) indican que la magnitud del traslado del impuesto al precio de venta puede variar por marca, tipo de comercio, poder de mercado de los productores, la distancia a comercios que tienen menores precios y el tipo de impuesto a los cigarros.

Los primeros estudios sobre incidencia bajo competencia imperfecta para la industria del tabaco se realizaron en la década de los 80's. Sobresalen los trabajos de Sumner (1981), con extensiones de Bulow y Pfeiderer (1983), y Sullivan (1985), Ashenfelter y Sullivan (1987), y Harris (1987). 
Los trabajos de Barnett et al. (1995) y Delipalla y O’Donnell (2001), proponen modelos estructurales de la industria para analizar la incidencia impositiva. Estos modelos abordan la incidencia, expresando por separado las condiciones asociadas con la estructura de mercado de la industria, la demanda del producto y la estructura de costos de las empresas participantes.

En cuanto a la estructura de datos, los estudios de Sung et al. (1994) y Sullivan y Dutkowsky (2012), utilizan datos que combinan series de tiempo con corte transversal. Los trabajos más recientes, han estimado la incidencia de impuestos al tabaco empleando microdatos. En algunos casos, los datos provienen de encuestas a consumidores (Xu et al., 2014; DeCicca et al., 2013; Pesko et al., 2013), datos de escáner a nivel de UPC (Wang et al., 2015; Chiou y Muehlegger, 2014; Harding et al., 2012), o bien datos por establecimiento comercial, a través del tiempo y para distintas marcas de cigarrillos (Cevik, 2016; Brock et al., 2015; Gilmore et al., 2013; Espinosa y Evans, 2013; Sullivan y Dutkowsky, 2012; Hanson y Sullivan, 2009). Solo para efectos de ilustración, Harding et al. (2012) es de los estudios sobre incidencia impositiva en la industria del tabaco que ha empleado datos más detallados. Se trata de datos escaneados de Nielsen sobre las compras de hogares a nivel UPC para 2006 y 2007. Los datos ofrecen detalle sobre el tipo de producto comprado y la ubicación de la compra, con lo que se pueden ubicar tanto las características de la zona como las sociodemográficas de los consumidores. Se cubren 160969 transacciones realizadas por 10784 hogares en 48 estados y el Distrito de Columbia en USA.

Wang et al. (2015), también utilizan datos de escáner al nivel de tiendas de conveniencia (1 865) que corresponden a 560 condados de 48 estados de USA en el período 2011-2012. Dado que los datos son al nivel de UPC, en ese estudio fue posible controlar las variaciones de los precios usando las características de los productos y analizar cómo interactúa la incidencia impositiva con la heterogeneidad de los productos. Wang et al. (2015) definen un modelo en forma reducida en que el precio es una función del impuesto, controlando por los atributos del producto, las condiciones económicas sociales y demográficas de los condados (porcentaje de hombres, negros, asiáticos e hispanos en la población; ingreso per cápita; tasa de desempleo; y porcentaje de graduados de preparatoria y universidad), además de incorporar efectos fijos de los condados. En efecto, entre los atributos del producto incluyen si el cigarrillo es regular, mentolado o de otro sabor; si es con filtro o sin filtro; si es regular, light o ultra light; si se trata de una marca Premium o de descuento; y si el empaque es cajetilla de 20 cigarrillos o paquete de diez cajetillas. Los autores encontraron que, en promedio, un aumento de $\$ 1$ en el impuesto a los cigarrillos, traería como consecuencia un aumento en el precio de más de $\$ 1$, que puede ir de 5 a 19 centavos por cajetilla de cigarrillos. Este efecto, es diferente en función de las distintas clases de cigarrillos. Encuentran que el traslado del impuesto para cigarrillos vendidos en cartón es 13 centavos mayor al de cajetillas; mientras que los cigarros Premium tienen una carga mayor en 7 centavos que las marcas de descuento. 
En una industria altamente concentrada, como lo es la del tabaco, el traslado al precio por encima del monto del impuesto sugiere la existencia de poder de mercado y conducta estratégica de las firmas en la industria.

Xu et al. (2014) y diferentes tratados sobre economía del tabaco (U.S. National Cancer Institute y World Health Organization, 2016; Samet y Yoon, 2010; Chaloupka y Warner, 2001; Chaloupka et al., 2000), indican que el traslado de los impuestos a los precios pagados por los consumidores depende de una serie de factores: el tipo de impuesto, la naturaleza adictiva del tabaco, la estructura de mercado de la industria, la diversidad de marcas y segmentación de mercados propias de la industria, la magnitud del comercio ilegal de tabaco y la magnitud de la elusión de impuestos.

Nuestro artículo se enmarca en los estudios sobre incidencia de impuestos al tabaco que utilizan datos agregados en serie de tiempo, tales como Galbraith y Kaiserman (1997), Stehr (2005), Keeler (1993), Hu et al. (1994), Hu et al. (1995), y más recientemente Cetin (2017), así como aquellos que especifican una forma reducida para estimar la incidencia de impuestos en la industria del tabaco (Delipalla y O'Donnell, 2001; Keeler et al., 1996; Harding et al., 2012: Wang et al., 2015; DeCicca et al., 2013; Pesko et al., 2013).

Como lo anotamos en la introducción, no tenemos conocimiento de otro estudio que estime la incidencia impositiva en la industria del tabaco mexicana.

\section{Metodología}

Para analizar la incidencia de los impuestos al tabaco en México, especificamos un modelo en forma reducida y lo estimamos usando 282 observaciones mensuales, de enero 1994 a junio 2017. Con base en las formas reducidas, que se utilizan en la gran mayoría de la literatura relacionada (Poterba, 1996; Besley y Rosen, 1999; Delipalla y O'Donnell, 2001; Keeler et al., 1996; Harding et al., 2012; Wang et al., 2015; DeCicca et al., 2013; Pesko et al., 2013), definimos el modelo para explicar las variaciones mensuales en los precios de los productos de tabaco $(\mathrm{Pt})$ como:

$$
P_{t}=\beta_{1}+\beta_{2} \tau_{t}+x_{t}^{\prime} \delta+\varepsilon_{t} .
$$

Donde $\tau$ representa los impuestos a productos de tabaco en México (IEPS ad-valorem y IEPS específico) y X es un vector que incluye variables para controlar por los determinantes de la demanda de tabaco (PIBPC, RLEY, RPIC), los costos de producción de productos de tabaco (IPP, SAL), la estructura de mercado de la industria de tabaco (HHI), así como la tendencia (T) y estacionalidad (EST) que pueda observar la variable Pt. $\varepsilon$ representa el término del error. La descripción de las variables, estadística descriptiva y las fuentes de información se presentan en la tabla 2. 


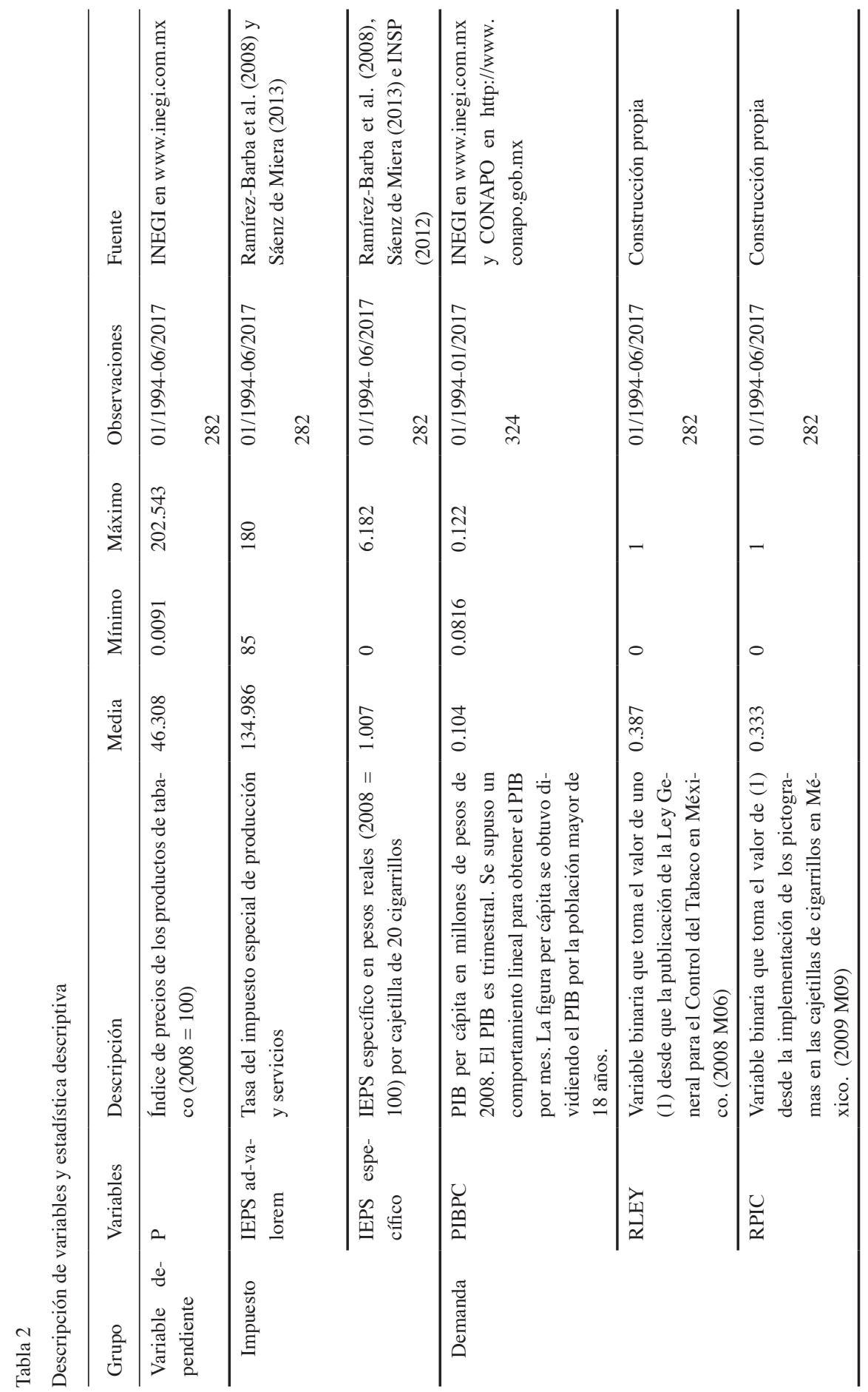




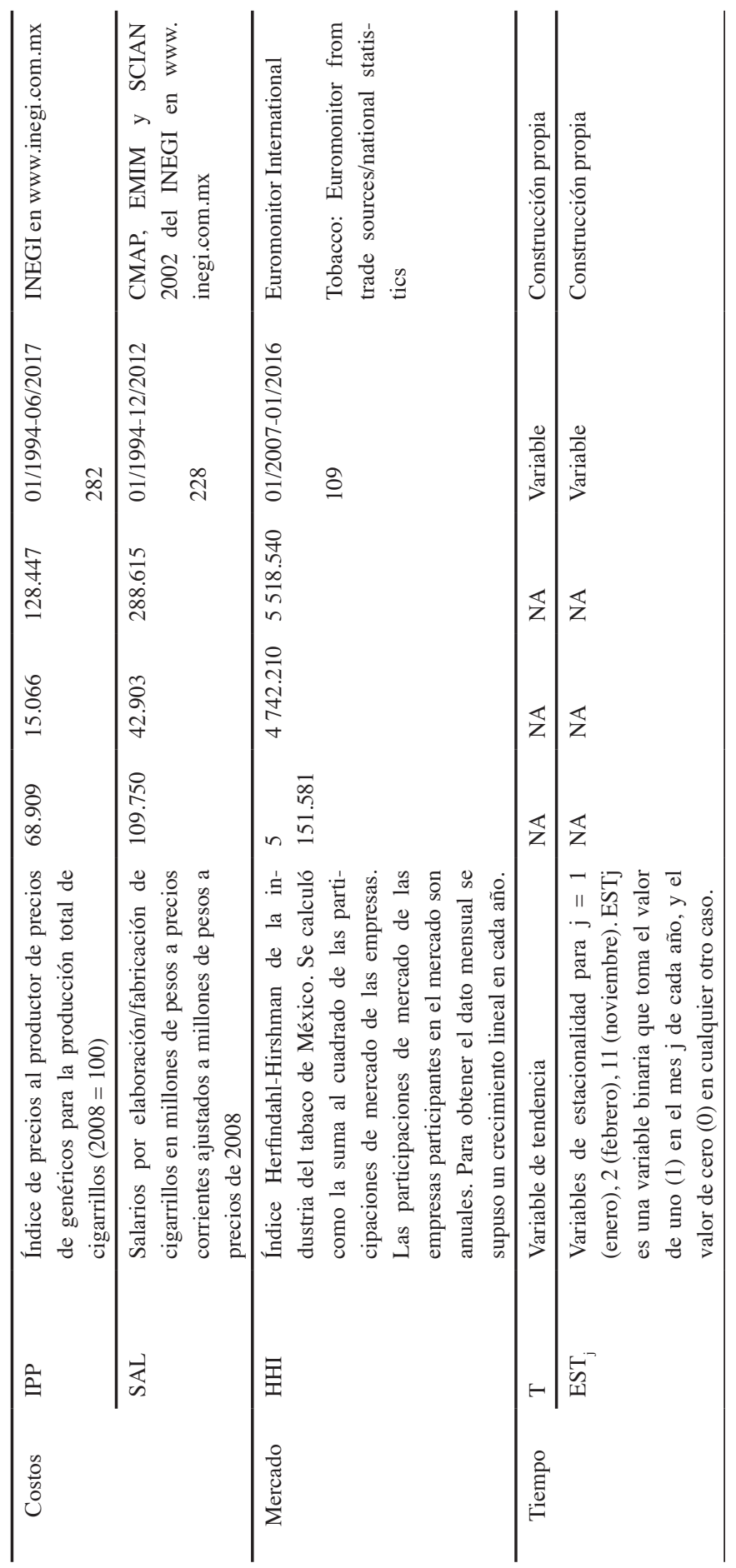

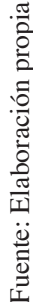


La variable dependiente es el índice mensual de precios de los productos de tabaco. El impuesto al tabaco lo medimos con la tasa del IEPS y con el monto específico de este impuesto, por cajetilla de 20 cigarrillos, que se introdujo en enero de 2010. Las variables independientes, asociadas con la demanda de tabaco son: el Producto Interno Bruto per cápita (PIBPC) y dos variables indicativas de las regulaciones que pudieron influir en la demanda (La Ley General para el Control del Tabaco en México (RLEY) y la entrada en vigor de las advertencias sanitarias con pictogramas en las cajetillas de cigarrillos (RPIC)).

Las variables que usamos para controlar por los cambios en los costos de producción de los productos de tabaco son: el índice de precios al productor de cigarrillos (IPP) y los salarios relacionados con la fabricación de cigarrillos (SAL).

Para medir la estructura de mercado en la industria del tabaco, calculamos el índice de Herfindal - Hirshmann (HHI) a partir de las participaciones de mercado anuales de las empresas que conforman esa industria.

Finalmente, incluimos como controles una variable de tendencia $(\mathrm{T})$ y variables binarias asociadas a cada mes del año, para reconocer la posible estacionalidad en el índice de precios de productos de tabaco. Tal como lo indica Greene (2008, p. 108), la inclusión explícita de las variables temporales es una forma de desestacionalizar los datos.

Todos los datos que usamos en las estimaciones son publicados con una frecuencia mensual, excepto las participaciones de mercado de las empresas que componen la industria, que empleamos para calcular la variable HHI, y el PIB. Las participaciones de mercado tienen una frecuencia anual y el PIB tiene una frecuencia trimestral. En ambos casos, y siguiendo Gaynor y Kirkpatrick (1994), realizamos una proyección lineal en cada período para calcular los datos mensuales.

En función de la disponibilidad de datos para aproximar las variables independientes y buscando valorar la consistencia de nuestros resultados, definimos tanto la estrategia de estimación como los diferentes modelos empíricos. En particular, consideramos dos criterios en nuestras estimaciones. En primera instancia, consideramos la disponibilidad de datos en el período bajo estudio. Contamos con observaciones desde 01/1994 hasta 06/2017 para todas las variables, excepto SAL, disponible de 01/1994 a 12/2012, y HHI con observaciones disponibles de 01/2007 a 01/2016. En ese caso, estimamos cuatro modelos, incluyendo todas las variables independientes, y otros tres alternando las variables SAL y HHI.

El segundo criterio, fueron las variables que integran cada grupo (demanda, costos, estructura de mercado y tiempo). Estimamos la relación entre el precio de los productos de tabaco y el impuesto al tabaco, incluyendo en forma secuencial las variables independientes de cada uno de los grupos. Además, especificamos modelos lineales y con las variables en logaritmos naturales. El modelo lineal lo especificamos de la siguiente manera: 


$$
\begin{aligned}
P_{t}= & \beta_{0}+\beta_{1} \text { IEPS ad-valorem }+\beta_{2} \text { IEPS específico }+\delta_{1} \text { PIBPC }+\delta_{2} \text { RLEY }+\delta_{3} \text { RPIC } \\
& +\delta_{4} \text { IPP }+\delta_{5} \text { SAL }+\delta_{6} \text { HHI }+\delta_{7} \mathrm{~T}+\sum_{j=1}^{11} \delta_{7+j} \text { EST }_{j}+\varepsilon_{t} .
\end{aligned}
$$

El modelo logarítmico, lo especificamos como:

$$
\begin{aligned}
& P_{t}=\beta_{0}+\beta_{1} \text { IEPS ad-valorem }+\beta_{2} \ln (\text { IEPS específico })+\delta_{1} \ln (\text { PIBPC })+\delta_{2} \text { RLEY } \\
& +\delta_{3} \text { RPIC }+\delta_{4} \ln (\mathrm{IPP})+\delta_{5} \ln (\mathrm{SAL})+\delta_{6} \ln (\mathrm{HHI})+\delta_{7} \mathrm{~T}+\sum_{\mathrm{j}=1}^{11} \delta_{7+\mathrm{j}} \mathrm{EST}_{\mathrm{j}}+\varepsilon_{\mathrm{t}}
\end{aligned}
$$

El método de estimación fue mínimos cuadrados ordinarios (MCO), usando la corrección Newey-West, que estima errores robustos ante la presencia de autocorrelación y/o heteroscedasticidad. Usamos el VIF (variance inflation factor) para detectar multicolinealidad. Como regla general, un VIF mayor a 10 es indicio de multicolinealidad severa, que requiere de alguna medida correctiva. ${ }^{8}$

\section{Resultados}

Las tablas 3 a 5 contienen los resultados de las estimaciones. En la tabla 3 aparecen los modelos estimados variando el número de observaciones, al alternar las variables SAL y HHI. Como se puede apreciar, los resultados muestran evidencia de multicolinealidad severa en todos los modelos estimados. En particular, las variables T e IPP observan VIF's que varían de 253 a 726, para la primera variable, y entre 233 y 780, para la segunda. La presencia de multicolinealidad puede provocar cambios bruscos en los valores y signos de los parámetros estimados, tal como se puede apreciar, por ejemplo, entre los modelos 2 y 3 en la tabla 3. Igualmente, la multicolinealidad puede movernos a no rechazar hipótesis de significancia estadística, que al corregir el problema deban ser rechazadas. Ambas situaciones, nos llevarían a conclusiones erróneas con relación al efecto del impuesto sobre el precio de los productos del tabaco.

Para tratar de corregir este problema, eliminamos la variable de tendencia (T) en la estimación de los modelos. Aunque el problema se reduce, aún persiste. El índice de precios

\footnotetext{
${ }^{8}$ Kutner et al. (2004) y también en 2.4 - Detecting Multicollinearity Using Variance Inflation Factor. https://onlinecourses.science.psu.edu/ stat501/ node/ 347
} 
al productor (IPP) y el índice de Herfindahl - Hirshman (HHI) parecen ser las variables que causan el problema. Otra alternativa que aplicamos para aminorar la multicolinealidad, fue estimar los modelos convirtiendo las variables en logaritmos naturales, aunque tampoco ayudó a aminorar el problema de multicolinealidad. De nuevo, las variables logarítmicas de IPP y HHI, entre otras, siguieron causando este problema.

Siguiendo la estrategia de incluir las variables independientes en bloques, ayuda a obtener algunas estimaciones que muestran un grado de multicolinealidad aceptable, para efectos de hacer inferencia estadística e interpretar los parámetros estimados (tablas 4 y 5). En dichas estimaciones, tanto la tasa de IEPS ad-valorem como el IEPS específico muestran una relación directa, que además es estadísticamente significativa en los modelos 3, 4 y 5 de la tabla 4. De acuerdo con el parámetro estimado de la variable IEPS específico, en el modelo 3 de la tabla 4 , un aumento de $\$ 1$ peso por cajetilla en el componente específico del IEPS incrementaría el índice de precios de productos de tabaco en 6.1 puntos, lo que equivale a un incremento de $3 \%$ tomando como base el dato de junio de 2017 (202.5).

El modelo logarítmico 3, en la tabla 5, indica que el índice de precios de productos de tabaco es inelástico con respecto tanto al IEPS específico, como al IEPS ad-valorem. En efecto, la elasticidad estimada con respecto al IEPS ad-valorem es 0.003 y con respecto al IEPS específico es 0.113 . Usando este modelo, un incremento de $20.4 \%$ en el componente específico del impuesto provocaría un incremento en el índice de precios de productos de tabaco de $2.3 \%$. Con relación al componente ad-valorem del IEPS, si la tasa aumentara 20 puntos porcentuales, entonces los precios al consumidor de productos de tabaco aumentarían un $0.05 \%$. Este resultado es similar a los reportados en la literatura relacionada. En particular, Sumner y Ward (1981), Harding et al. (2012), Pesko et al. (2013), Chiou y Muehlegger (2014), y Cevik (2016) encontraron que los precios de los cigarrillos aumentan menos que el impuesto.

Con relación a las variables de control, se puede apreciar en la tabla 4, que la variable binaria del modelo lineal para indicar la vigencia de la Ley General para el Control del Tabaco (RLEY), muestra una relación directa con el índice de precios de productos de tabaco, que además es estadísticamente significativa en los modelos 2 y 3 . Parecería que las diversas medidas regulatorias, implicadas en la Ley, trajeron como consecuencia que aumentaran los precios del tabaco.

Los resultados en la tabla 4 , también nos muestran que la variable indicativa de la regulación de etiquetado en las cajetillas de cigarros, a través de pictogramas (RPIC), observa una relación inversa y estadísticamente significativa en los modelos 3, 4 y 5. Toda vez que esa regulación pudo haber reducido la demanda de productos de tabaco, tal como se infiere de Thrasher et al. (2007) y Thrasher et al. (2012), y tal como se demuestra en Ibarra Salazar et al. (2019), la industria pudo haber respondido reduciendo los precios para contrarrestar este efecto. Si bien esto se deduce de la estimación del modelo lineal, los resultados de la 
estimación del modelo en logaritmos no confirman esos resultados. Tal como se aprecia en la tabla 5, en los modelos 3, 4 y 5, no es posible rechazar la hipótesis de que los parámetros de las variables asociadas con la regulación de la industria son diferentes de cero.

De acuerdo a la hipótesis precio - concentración de mercado (Bresnahan, 1989), se esperaría que mayor concentración en la industria condujeran a mayores precios. Los resultados en la tabla 4, nos indican que el HHI no es estadísticamente significativo, aunque como anotamos arriba, la variable muestra multicolinealidad elevada. Por esa razón no es posible determinar cuál es el papel que juega la estructura de mercado en la evolución de los precios de los productos de tabaco.

Finalmente, y como se esperaría, el índice de precios a los productores de cigarrillos (IPP) muestra también una relación directa y significativa desde el punto de vista estadístico con los precios de productos de tabaco.

En resumen, los resultados de las estimaciones muestran que:

- Tanto la tasa de IEPS como el IEPS específico guardan una relación directa y estadísticamente significativa con el índice de precios de productos de tabaco;

- El índice de precios de productos de tabaco es poco sensible a cambios en el IEPS ad-valorem y el IEPS específico.

- No es posible determinar en forma consistente el efecto que sobre los precios de productos de tabaco tienen la estructura de mercado y las medidas regulatorias consideradas en el estudio (Ley General para el Control del Tabaco y la regulación de advertencias sanitarias con pictogramas).

- La elasticidad de los precios de productos de tabaco con respecto al índice de precios a los productores de cigarros es aproximadamente igual a uno. 


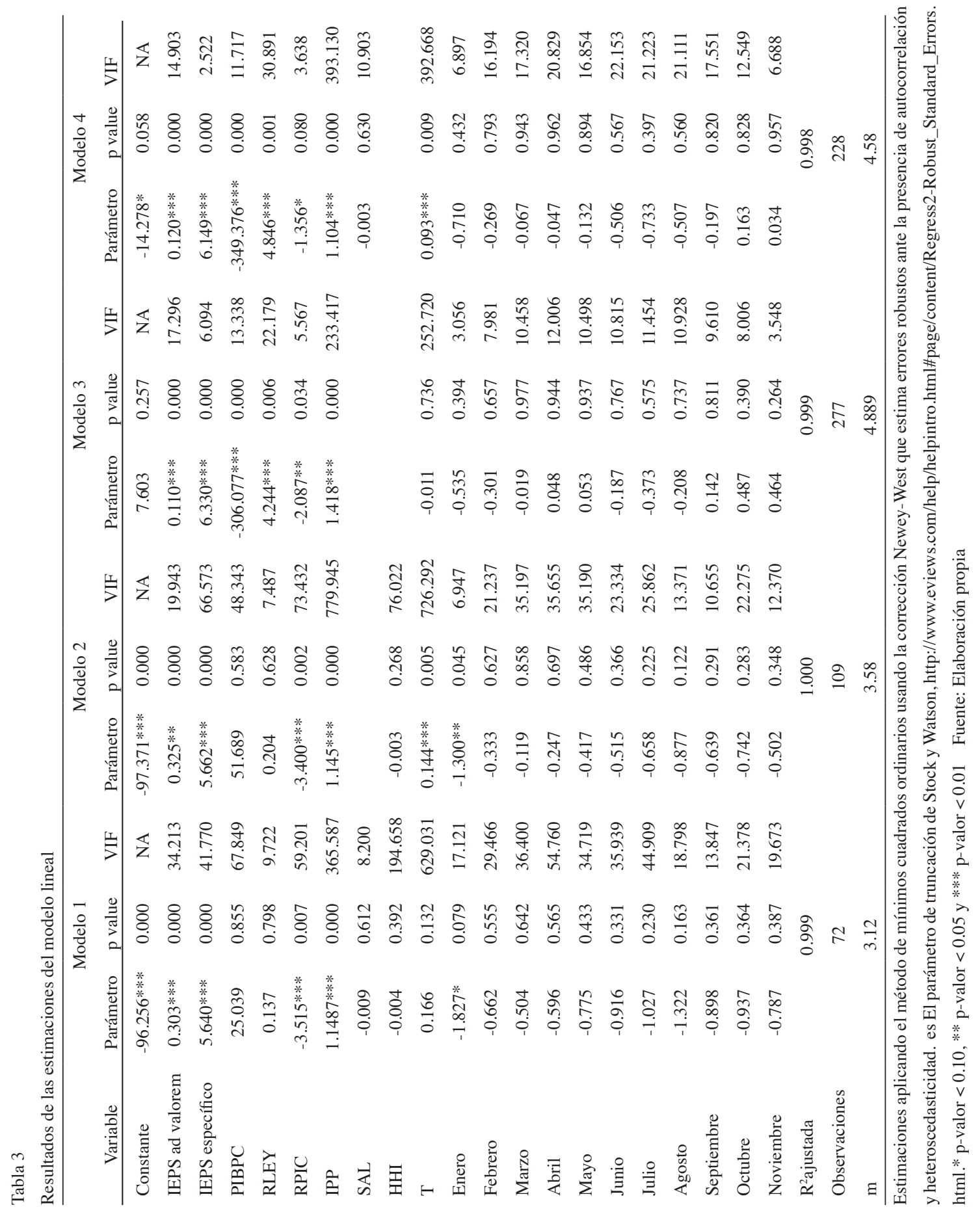




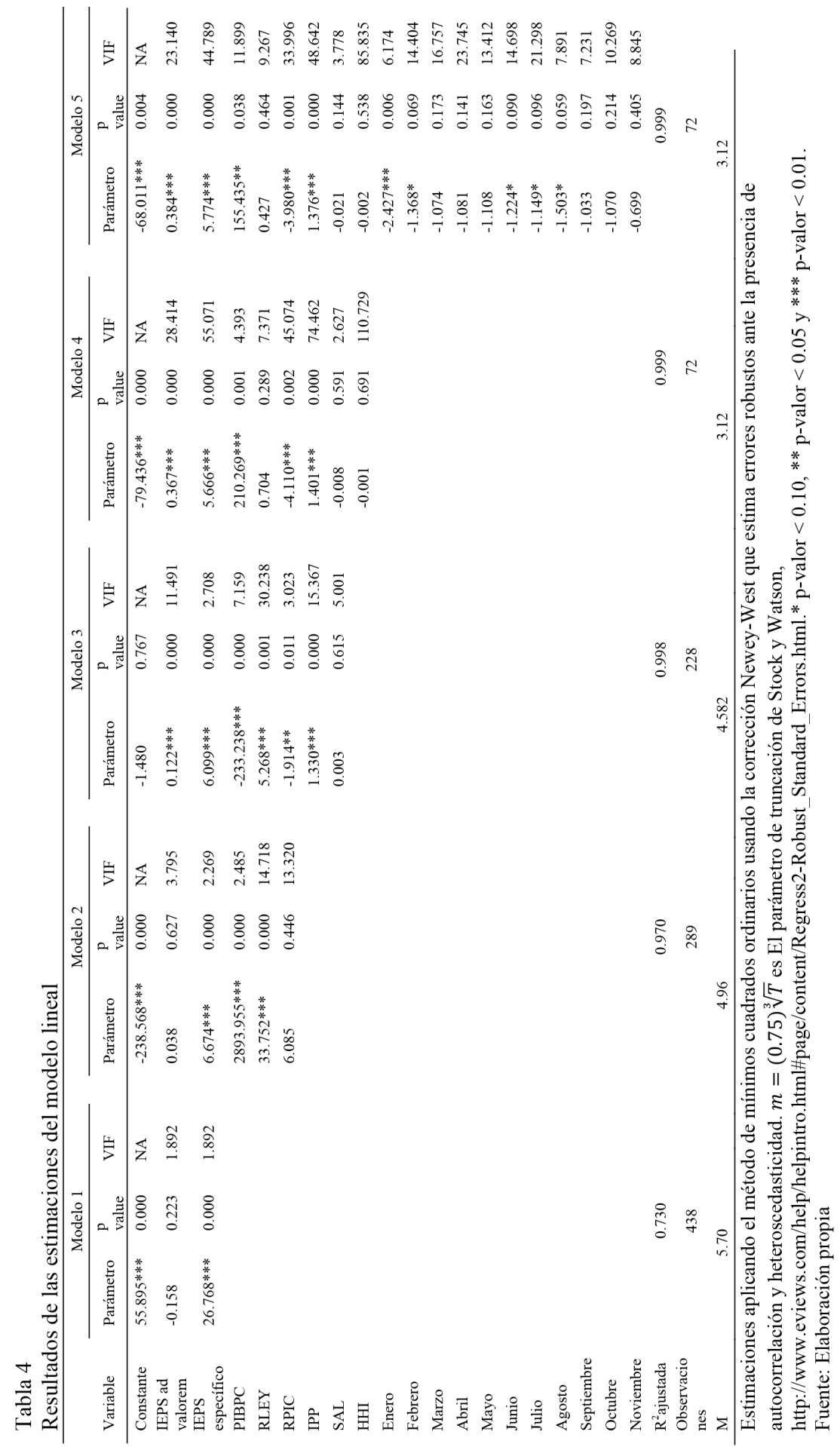




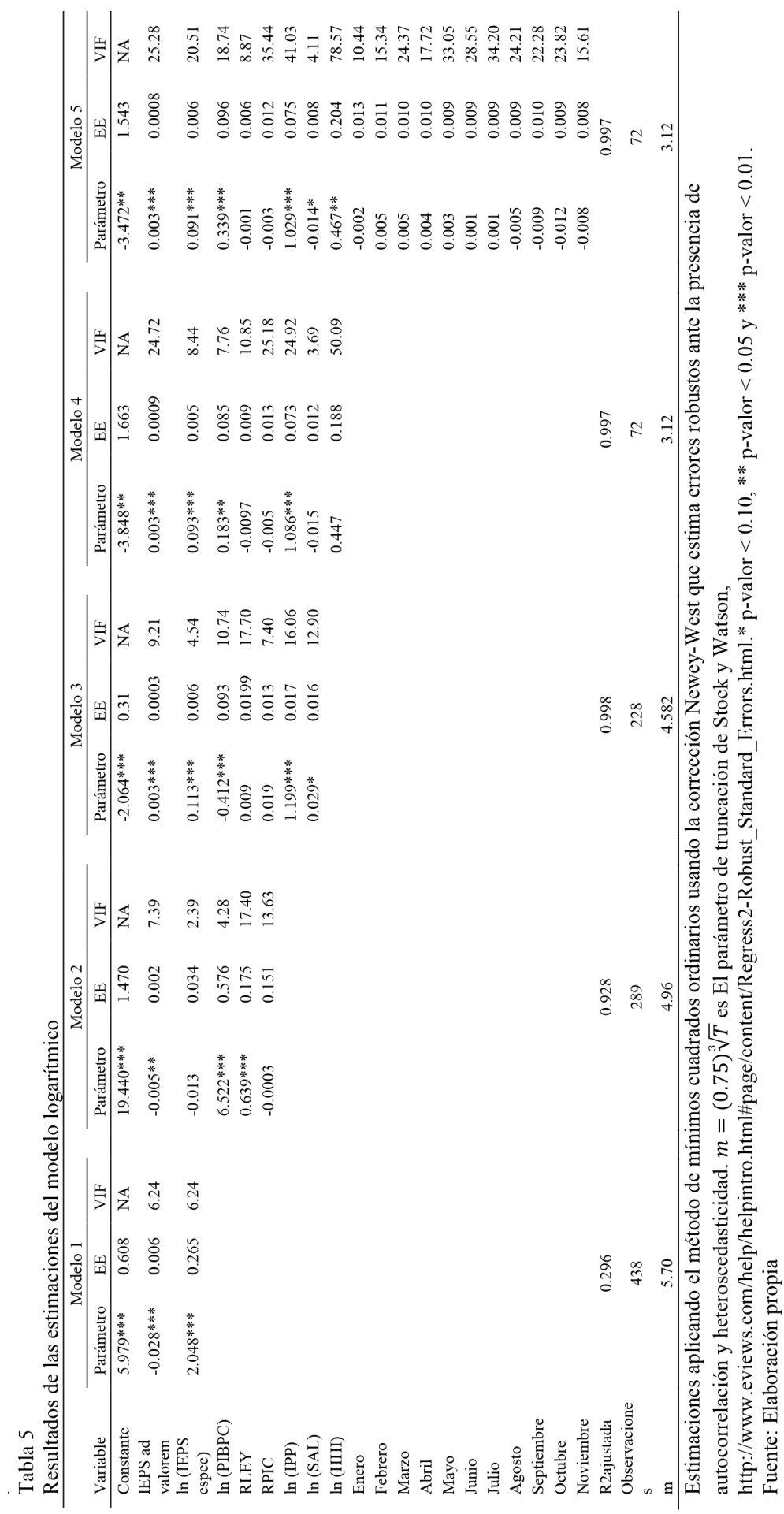




\section{Conclusiones}

En este artículo hemos propuesto correlacionar el índice de precios de productos de tabaco, con el IEPS a través de una forma reducida que, además del impuesto a productos de tabaco, incluye como variables independientes a las determinantes de la demanda, de los costos de producción, la estructura de mercado, así como variables para reconocer la tendencia y estacionalidad. El IEPS lo medimos con la tasa de IEPS ad-valorem y con el monto del impuesto específico por cajetilla de 20 cigarrillos.

Para aminorar el problema de multicolinealidad, estimamos los modelos variando el número de observaciones, de acuerdo con la disponibilidad de datos de las variables independientes, incluyendo bloques de variables independientes y aplicando diferentes formas funcionales a la relación causal de interés.

Los resultados con un grado de multicolinealidad aceptable, muestran evidencia de que tanto el IEPS ad-valorem como el específico guardan una relación directa y estadísticamente significativa con el índice de precios de productos de tabaco. En particular, el modelo lineal indica que un aumento de $\$ 1$ peso en el componente específico por cajetilla, que representaría un 20.4\% del componente específico real a junio de 2017, incrementaría el índice de precios a productos de tabaco en 6.1 puntos, lo que equivale a un $3 \%$ tomando como base el dato de junio de 2017 (202.5). Usando el modelo en logaritmos, un incremento de $20.4 \%$ en el componente específico del impuesto provocaría un incremento en el índice de precios de productos de tabaco de $2.3 \%$. Con relación al componente ad-valorem del IEPS, si la tasa aumentara 20 puntos porcentuales, entonces los precios al consumidor de productos de tabaco aumentarían un $0.05 \%$.

En términos de incidencia impositiva, estos resultados nos indican que los incrementos en los impuestos no se han traducido en incrementos de la misma magnitud en el índice de precios de productos de tabaco. Una de las condiciones, para que los impuestos puedan reducir el tabaquismo, es que sus ajustes al alza sean trasladados a los precios que pagan los consumidores, para que de esa forma se reduzca la demanda. De acuerdo a nuestros resultados, si bien encontramos una relación directa entre los impuestos al tabaco y el índice de precios de productos de tabaco, la magnitud de aumentos en los precios es menor que el incremento en el IEPS. Nuestro resultado resta validez a los estudios que han estimado el efecto de ajustes en los impuestos sobre los precios de cigarrillos y la recaudación de impuestos al tabaco, ya que han supuesto que los ajustes en impuestos son trasladados a los consumidores a través de los precios.

De acuerdo con la literatura sobre incidencia impositiva de la industria del tabaco, nuestro resultado es similar al de Sumner y Ward (1981), Harding et al. (2012), Pesko et al. (2013), Chiou y Muehlegger (2014), y Cevik (2016) en el sentido que, al aumentar el impuesto sobre 
productos de tabaco, los precios a los consumidores aumentan en menor proporción.

Tal como hemos comentado arriba, Xu et al (2014) indican que la magnitud del traslado del impuesto al precio de venta puede variar por marca, tipo de comercio, poder de mercado de los productores, la distancia a comercios que tienen menores precios y el tipo de impuesto a los cigarros. En México, la contención del índice de precios a productos de tabaco ante ajustes en los impuestos se puede explicar por la tendencia a la baja de la demanda del tabaco, en presencia del comercio ilegal de tabaco.

La incidencia analizada en este artículo es con respecto al índice de precios de productos de tabaco. Sería interesante averiguar si el efecto de modificaciones en los impuestos a los productos de tabaco es diferente en función de características regionales, la estructura de mercado de la industria, y los tipos de cigarros que se comercializan en México.

\section{Referencias}

Ashenfelter, O., \& Sullivan, D. (1987). Tests of market structure: An application to the cigarette industry. The Journal of Industrial Economics, 35 (4), 483-498. https://doi. org/10.2307/2098584.

Barnett, P. G., Keeler, T. E., \& Hu, T. W. (1995). Oligopoly structure and the incidence of cigarette excise taxes. Journal of Public Economics, 57 (3), 457-470. https://doi. org/10.1016/0047-2727(95)80006-U.

Besley, T. J., \& Rosen, H. S. (1999). Sales taxes and prices: An empirical analysis. National Tax Journal, 52 (2), 157. https://www.jstor.org/stable/41789387.

Bresnahan, T. (1989). Empirical studies of industries with market power. En R. Schmalensee \& R. Willig (Ed.), Handbook of Industrial Organization. (pp. 1011-1057). Amsterdam: North-Holland. https://doi.org/10.1016/S1573-448X(89)02005-4.

Brock, B., Choi, K., Boyle, R. G., Moilanen, M., \& Schillo, B. A. (2015). Tobacco product prices before and after a statewide tobacco tax increase. Tobacco Control, 25 (2), 166173. http://dx.doi.org/10.1136/tobaccocontrol-2014-052018.

Bulow, J. \& Pfleiderer, P. (1983). A note on the effect of cost changes on prices. Journal of Political Economy, 91 (1), 182-185. https://doi.org/10.1086/261135.

CEFP (2002). Estudio económico y fiscal de la industria tabacalera de México: 1990-2001. CEFP/038/2002. Disponible en: http://www.cefp.gob.mx/intr/edocumentos/pdf/cefp/ cefp 0382002.pdf. Consultado: 12/02/2018.

CEFP (2018). El tabaquismo y su efecto en las finanzas Públicas: 2007-2017. Notacefp/004/2018. Disponible en: http://www.cefp.gob.mx/publicaciones/nota/2018/notacefp0042018.pdf. Consultado: 12/02/2018.

Cetin, T. (2017). The effect of taxation and regulation on cigarette smoking: fresh evidence from Turkey. Health Policy, 121 (12), 1288-1295. https://doi.org/10.1016/j.healthpol.2017.09.015. 
Cevik, M. S. (2016). Smoke Screen: Estimating the Tax Pass-Through to Cigarette Prices in Pakistan. International Monetary Fund. Disponible en: https://www.imf.org/en/Publications/WP/Issues/2016/12/31/Smoke-Screen-Estimating-the-Tax-Pass-Through-to-Cigarette-Prices-in-Pakistan-44202. Consultado: 15/03/2018.

Chaloupka F., Hu, T., Warner, K., Jacobs, R., \& Yurekli, A. (2000). The taxation of tobacco products. En P. Jha \& F. Chaloupka (Ed.). Tobacco control in developing countries. (pp. 237-272). Oxford: Oxford University Press.

Chaloupka, F., \& Warner, K. (2001). The economics of smoking. En A. Culyer \& J. Newhouse (Ed.). Handbook of health economics. (pp. 1539-1627). New York: Elsevier.

Chiou, L., \& Muehlegger, E. (2014). Consumer response to cigarette excise tax changes. National Tax Journal, 67 (3), 621-650. http://doi.org/10.17310/ntj.2014.3.05.

COFEMER. (2012). Tabaquismo en México: análisis y recomendaciones de mejora regulatoria. México: COFEMER.

CONADIC (2011). Programa contra el Tabaquismo: Actualización 2011-2012. Disponible en: http://www.conadic.salud.gob.mx/pdfs/publicaciones/tabaquismo.pdf. Consultado: 15/04/2018.

DeCicca, P., Kenkel, D., \& Liu, F. (2013). Who pays cigarette taxes? The impact of consumer price search. Review of Economics and Statistics, 95 (2), 516-529. https://doi. org/10.1162/REST_a_00303.

Delipalla, S., \& O’Donnell, O. (2001). Estimating tax incidence, market power and market conduct: The European cigarette industry. International Journal of Industrial Organization, 19 (6), 885-908. https://doi.org/10.1016/S0167-7187(99)00057-0.

Espinosa, J., \& Evans, W. N. (2013). Excise taxes, tax incidence, and the flight to quality: Evidence from scanner data. Public Finance Review, 41 (2), 147-176. https://doi. org/10.1177/1091142112460724.

Galbraith, J., \& Kaiserman, M. (1997). Taxation, smuggling, and the demand for cigarettes in Canada: evidence from time series data. Journal of Health Economics, 16 (3), 287301. https://doi.org/10.1016/S0167-6296(96)00525-5.

Gaynor, P., \& Kirkpatrick, R. (1994). Introduction to time-series modeling and forecasting in business and economics. New York: McGraw-Hill.

Gilmore, A. B., Tavakoly, B., Taylor, G., \& Reed, H. (2013). Understanding tobacco industry pricing strategy and whether it undermines tobacco tax policy: the example of the UK cigarette market. Addiction, 108 (7), 1317-1326. https://doi.org/10.1111/add.12159.

Greene, W. (2008). Econometric analysis. (6th Ed.). Upper Saddle River: Pearson - Prentice Hall.

Guerrero, C., Reynales, L., Jiménez, J., Karam, R., Maldonado, C., \& Camacho, R. (2012). Costos por ausentismo laboral atribuibles al consumo de tabaco en el Instituto Mexicano del Seguro Social y en México, 2006-2009. Salud Pública de México, 54 (3), 233-241. http://saludpublica.mx/index.php/spm/article/view/7134. 
Hanson, A., \& Sullivan, R. (2009). The incidence of tobacco taxation: evidence from geographic micro-level data. National Tax Journal, 62 (4), 677-698. https://doi.org/10.17310/ ntj.2009.4.05.

Harding, M., Leibtag, E., \& Lovenheim, M. F. (2012). The heterogeneous geographic and socioeconomic incidence of cigarette taxes: Evidence from Nielsen homescan data. American Economic Journal: Economic Policy, 4 (4), 169-198. https://doi.org/10.1257/ pol.4.4.169.

Harris, J. E. (1987). The 1983 increase in the federal cigarette excise tax. Tax Policy and the Economy, 1, 87-111. https://www.jstor.org/stable/20061764.

Hu, T., Bai, J., Keeler, T., Barnett, P., \& Sung, H. (1994). The impact of California proposition 99, a major anti-smoking law, on cigarette consumption. Journal of Public Health Policy, 15 (1), 26-36. https://doi.org/10.2307/3342605.

Hu, T., Keeler T., Sung, H. \& Barnett, P. (1995). The impact of California antismoking legislation on cigarette sales, consumption, and prices. Tobacco Control, 4 (1), S34-S38. http://dx .doi.org/10.1136/tc.4.suppl1.S34.

Ibarra Salazar, J., González Caloca, C., Núñez Gómez, S., Ramírez García, M., Rodríguez Nuncio, M., \& Santos González, C. (2019). La regulación de etiquetado en la industria del tabaco de México: Efecto de los pictogramas en la demanda de tabaco. Economía Teoría y Práctica, 27 (51), 73-104. http://dx.doi.org/10.24275/ETYPUAM/NE/512019/ Ibarra.

Instituto Nacional de Salud Pública, INSP (2011). Encuesta Nacional de Adicciones 2011. Tabaco. Disponible en: https://www.insp.mx/produccion-editorial/publicaciones-anteriores-2010/2968-encuesta-nacional-de-adicciones-2011-tabaco.html. Consultado: 15/04/2018.

Instituto Nacional de Salud Pública, INSP (2012). México: historia acerca del éxito del impuesto al tabaco. Disponible en: http://tabaco.ficmexico.org/impuestos/. Consultado: 15/04/2018.

Jiménez, J., Saenz de Miera, B., Reynales, L., Waters, H., \& Hernández Ávila, M. (2008). The impact of taxation on tobacco consumption in Mexico. Tobacco Control, 17 (1), 105110. http://dx .doi.org/10.1136/tc.2007.021030.

Keeler, T. (1993). Taxation, regulation, and addiction: a demand function for cigarettes based on time-series evidence. Journal of Health Economics, 12 (1), 1-18. https://doi. org/10.1016/0167-6296(93)90037-F.

Keeler, T. E., Hu, T. W., Barnett, P. G., Manning, W. G., \& Sung, H. Y. (1996). Do cigarette producers price-discriminate by state? An empirical analysis of local cigarette pricing and taxation. Journal of Health Economics, 15 (4), 499-512. https://doi.org/10.1016/ S0167-6296(96)00498-5.

Kuri, P., González, J., \& Hoy, M. (2006). Epidemiología del tabaquismo en México. Salud Pública de México, 48 (1), S91-S98. http://saludpublica.mx/index.php/spm/article/ view/4703. 
Kutner, M., Nachtsheim, C., \& Neter, J. (2004). Applied linear regression models. (4th Ed.). New York: McGraw-Hill - Irwin.

Madrazo Lajous, A., \& Guerrero Alcántara, A. (2012). Estrategias de la industria tabacalera en México para interferir en las políticas de control del tabaco. Salud Pública de México, 54 (3), 315-322. http://saludpublica.mx/index.php/spm/article/view/7143.

Meneses-González, F., Márquez-Serrano, M., Sepúlveda-Amor, J., \& Hernández-Avila, M. (2002). The tobacco industry in Mexico. Salud Publica de México, 44 (1), S161-S169. https://www.scielosp.org/pdf/spm/2002.v44suppl1/s161-s169.

Ochoa, E. (2013). Las OSC como agentes clave en el proceso de las políticas públicas. Estudio de caso: el aumento a los impuestos de tabaco en México. En L. Reynales, J. Thrasher, E. Lazcano \& M. Hernández (Ed.). Política pública para el control del tabaco en México. (pp. 156-165). México: INSP.

Olivera-Chávez, R., Cermeño-Bazán, R., Sáenz de Miera, B., Jiménez-Ruíz, J., \& Reynales-Shigematsu, L. (2010). El efecto del precio del tabaco sobre el consumo: un análisis de datos agregados para México. Salud Pública de México, 52, S197-S205. https:// doi.org/10.1590/S0036-36342010000800015.

Pesko, M. F., Licht, A. S., \& Kruger, J. M. (2013). Cigarette price minimization strategies in the United States: price reductions and responsiveness to excise taxes. Nicotine \& Tobacco Research, 15 (11), 1858-1866. https://doi.org/10.1093/ntr/ntt068.

Poterba, J. M. (1996). Retail price reactions to changes in state and local sales taxes. National Tax Journal, 49 (2), 165-176. www.jstor.org/stable/41789195.

Ramírez-Barba, E., Saro-Boardmand, E., Vázaquez-Guerrero, A., \& Vázquez-Guerrero, M. (2008). Ley general para el control del tabaco en México. Salud Pública de México, 50, s372-s383. http://saludpublica.mx/index.php/spm/article/view/4850/7029.

Reynales, L., Levy, T., Méndez, I., Rojas, R., \& Lazcano, E: (2011). Encuesta Global de Tabaquismo en Adultos. México 2009. Cuernavaca: INSP, OPS.

Reynales, L., Rodríguez, R., Jiménez, J., Juárez, S., Castro, A., \& Hernández, M. (2006). Costos de la atención médica atribuibles al consumo de tabaco en el Instituto Mexicano del Seguro Social. Salud Pública de México, 48, S48-S64. http://saludpublica.mx/index. $\mathrm{php/spm/article/view/4699.}$

Reynales, L., Valdes. R., Rodríguez, R., Lazcano, E., \& Hernández, M. (2009). Encuesta de Tabaquismo en Jóvenes en México. Análisis descriptivo 2003, 2005, 2006, 2008. Cuernavaca, México: INSP.

Sáenz de Miera, B., Reynales, L., \& Jiménez, J. (2012). Tobacco consumption and household living standards in Mexico. $15^{\text {a }}$ Conferencia Mundial Tobacco or Health: Singapore. Sáenz de Miera, B. (2013). Impuestos al tabaco en México: análisis del período 2006-2012. En L. Reynales, J. Thrasher, E. Lazcano \& M. Hernández (Ed.) Política pública para el control del tabaco en México. (pp. 144-155). México: INSP. 
Sáenz de Miera, B., \& Iglesias, R. (2010). Impuestos para el control del tabaquismo: las experiencias de Brasil y México. Salud Pública de México, 52, s172-s185. http://saludpublica.mx/index.php/spm/article/view/4970/4818.

Sáenz de Miera, B., Guerrero, C., Zúñiga, J., \& Ruiz Velasco, S. (2013). Impuestos al tabaco y políticas para el control del tabaco. Resultados para México. México: Fundación Inter Americana del Corazón en México.

Samet, J., \& Yoon, S. (2010). Gender, women, and the tobacco epidemic. Manila: World Health Organization. Disponible en: http://www.who.int/tobacco/publications/gender/ women_tob_epidemic/en/. Consultado: 20/05/2018.

SSA (2011). SINAIS Sistema Nacional de Información en Salud, Tabla Dinámica de Defunciones, 1979ロ0009. Disponible en: http://www.sinais.salud.gob.mx/. Consultado: $15 / 04 / 2018$.

Stehr, M. (2005). Cigarette tax avoidance and evasion. Journal of Health Economics, 24 (2), 277-297. https://doi.org/10.1016/j.jhealeco.2004.08.005.

Sullivan, D. (1985). Testing hypotheses about firm behavior in the cigarette industry. Journal of Political Economy, 93 (3), 586-598. https://doi.org/10.1086/261317.

Sullivan, R. S., \& Dutkowsky, D. H. (2012). The effect of cigarette taxation on prices: an empirical analysis using local-level data. Public Finance Review, 40 (6), 687-711. https:// doi.org/10.1177/1091142112442742.

Sumner, D. (1981). Measurement of monopoly behavior: An application to the cigarette industry. Journal of Political Economy, 89 (5), 1010-1019. https://doi.org/10.1086/261017.

Sumner, D. A., \& Wohlgenant, M. K. (1985). Effects of an increase in the federal excise tax on cigarettes. American Journal of Agricultural Economics, 67 (2), 235-242. https://doi. org/ $10.2307 / 1240674$.

Sumner, M. T., \& Ward, R. (1981). Tax changes and cigarette prices. Journal of Political Economy, 89 (6), 1261-1265. https://doi.org/10.1086/261034.

Sung, H. Y., Hu, T. W., \& Keeler, T. E. (1994). Cigarette taxation and demand: an empirical model. Contemporary Economic Policy, 12 (3), 91-100. https://doi. org/10.1111/j.1465-7287.1994.tb00437.x.

Thrasher, J., Allen, B., Rousu, M., Anaya, R., Reynales, L., Arillo, E., \& Herández, M. (2007). Estimating the impact of different cigarette package warning label policies: the auction method. Addictive Behaviors, 32 (12), 2916-2925. https://doi.org/10.1016/j.addbeh.2007.05.018.

Thrasher, J., Pérez, R., Arillo, E., \& Barrientos, I. (2012). Impacto de las advertencias con pictogramas en las cajetillas de cigarros en México: resultados de una encuesta en fumadores de Guadalajara. Salud Pública de México, 54 (3), 254-263. http://saludpublica.mx/ index.php/spm/article/view/7136/9241 
U.S. National Cancer Institute, \& World Health Organization (2016). The economics of tobacco and tobacco control. National Cancer Institute Tobacco Control Monograph 21. NIH Publication No. 16-CA-8029A. Bethesda, MD: U.S. Department of Health and Human Services, National Institutes of Health, National Cancer Institute; and Geneva, CH: World Health Organization. Disponible en http://cancercontrol.cancer.gov/brp/tcrb/ monographs/21/index.html. Consultado: 15/04/2018.

Wang, X., Zheng, Y., Reed, M. R., \& Zhen, C. (2015). Cigarette tax pass-through by product characteristics: Evidence from Nielsen retail scanner data. Disponible en: SSRN: https:// ssrn.com/abstract=2686274. Consultado: 25/09/2017

Waters, H., Sáenz de Miera, B., Ross, H., \& Reynales Shigematsu, L. (2010). La economía del tabaco y los impuestos al tabaco en México. Paris: Unión Internacional contra la Tuberculosis y Enfermedades Respiratorias.

World Bank (1999). Curbing the epidemic: governments and the economics of tobacco control. The International Bank for Reconstruction and Development/ THE WORLD BANK.

World Health Organization (2015). WHO report on the global tobacco epidemic, 2015: Raising taxes on tobacco. Disponible en: http://www.who.int/tobacco/global_report/2015/ en/. Consultado: 15/04/2018

Xu, X., Malarcher, A., O'halloran, A., \& Kruger, J. (2014). Does every US smoker bear the same cigarette tax? Addiction, 109 (10), 1741-1749. https://doi.org/10.1111/add.12630. 